\title{
同地共栖三种鼠耳蝠食性差异及其生态位分化
}

\author{
胡开良 ${ }^{1}$, 杨 剑 $^{2}$, 谭梁静 ${ }^{2}$, 张礼标 ${ }^{3, *}$
}

(1. 临沂大学 生命科学学院, 山东 临沂 276000; 2. 广西师范大学 生命科学学院, 广西 桂林 $541004 ; 3$. 广东省昆虫研究所, 广东 广州 510260)

摘要： 2005 年 9-11 月在贵州省安龙县笃山乡暗河村, 分析了共栖同一山洞 3 种鼠耳蝠的形态特征和食性。

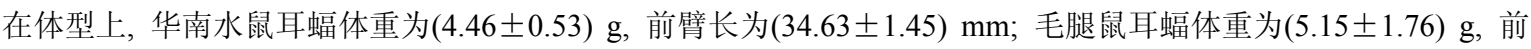

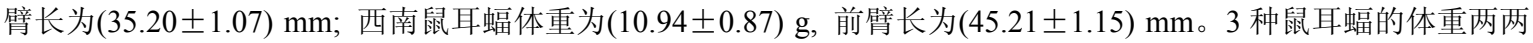
之间差异显著, 西南鼠耳蝠与另外 2 种鼠耳蝠的前臂长相比两两之间差异显著。在食物组成上, 华南水鼠耳蝠主 要捕食近水面活动的双翅目及其幼虫, 体积百分比和频次百分比分别为 $79.7 \%$ 和 $100 \%$; 毛腿鼠耳蝠主要捕食双翅 目和小型鞘翅目, 体积百分比分别占 $59.6 \%$ 和 $28.8 \%$, 频次百分比分别为 $91.3 \%$ 和 $80.1 \%$; 西南鼠耳蝠的食物组成 主要为近地面或在地表活动的鞘翅目步甲科和埋葬虫科昆虫，体积百分比和频次百分比分别为 $80.8 \%$ 和 $100 \% ; 3$ 种鼠耳蝠食物组成存在显著差异。结果表明, 同地共栖 3 种鼠耳蝠除了形态结构上出现差异, 食物组成也存在明 显的差异。据此，推测 3 种鼠耳蝠可能采取不同的捕食生境和捕食策略，从而导致捕食生态位分离，避免出现激烈 竞争, 使得 3 种近缘鼠耳蝠能够同地共栖。

关键词: 食性; 同地共栖种; 生态位; 鼠耳蝠

中图分类号: Q959.833; Q958.122.5 文献标志码：A 文章编号：0254-5853-(2012)02-0177-05

\section{Dietary differences and niche partitioning in three sympatric Myotis Species}

\author{
HU Kai-Liang ${ }^{1}$, YANG Jian ${ }^{2}$, TAN Liang-Jing ${ }^{2}$, ZHANG Li-Biao, ${ }^{3, *}$ \\ (1. College of Life Sciences, Linyi University, Linyi, Shandong 276000, China; 2. College of Life Sciences, Guangxi Normal University, \\ Guilin 541004, China; 3. Guangdong Entomological Institute, Guangzhou～510260, China)
}

\begin{abstract}
The morphology and diet characteristics of three Myotis species roosting in the same cave were studied in Anlong County, Guizhou from September to November 2005. The three mouse-eared bats were the Chinese water myotis (Myotis laniger) "body mass: (4.46 \pm 0.53$) \mathrm{g}$, forearm: $(34.63 \pm 1.45 \mathrm{~mm})$ ", fringed long-footed myotis (Myotis fimbriatus) "body mass: $(5.15 \pm 1.76) \mathrm{g}$, forearm: $(35.20 \pm 1.07) \mathrm{mm}$ " and szechwan myotis (Myotis altarium) "body mass: $(10.94 \pm 0.87) \mathrm{g}$, forearm: $(45.21 \pm 1.15) \mathrm{mm}$ ". There were significant differences in the body masses of the three species. The forearm length of $M$. altarium was significantly longer than the other two species. M. laniger preyed mostly on dipsters and their larvae (79.7\% in volume and 100\% in frequency, Diptera). M. fimbriatus preyed mostly on dipsters and small beetles $(59.6 \%$ and $91.3 \%$, Diptera; $28.8 \%$ and $80.1 \%$, Coleoptera). The prey of $M$. altarium was mostly ground-dwelling beetles $(80.8 \%$ and $100 \%$, Carabidae and Silphidae, Coleoptera). These dietary examinations indicate that the three species are highly adapted to different foraging habitats. Our work suggests that the spatial differences in foraging niches and trophic resource partitioning represent the major mechanism behind the levels of co-existence seen in this particular bat community.
\end{abstract}

Key words: Diet; Sympatric species; Niche; Myotis

鼠耳蝠属(Myotis)是翼手目(Chiroptera)蝙蝠科 (Vespertilionidae)中分布最广、种类最多的一个属; 我国已知共 16 种, 贵州分布有 6 种(Luo et al,
1993)。鼠耳蝠大部分为食虫蝙蝠，也有食鱼的种类， 如大足鼠耳蝠(Myotis ricketti), 但它同时也捕食昆 虫(Ma et al, 2003, 2005)。不同种类的鼠耳蝠常常共

收稿日期: 2011-06-21; 接受日期: 2011-12-12

基金项目: 广东省科学院人才基金(粤科优[2008]02 号); 北京林业大学 985 优势学科建设平台项目

*通信作者(Corresponding author), E-mail: zhanglb@gdei.gd.cn

第一作者简介: 男(1976-), 博士, 主要从事动物生态学研究。E-mail: hukailiang@lyu.edu.cn 
栖一地，这必然会引起对食物和空间资源的竞争。 Gaisler \& Bauerova (1985)认为不同种类的蝙蝠具有 不同的捕食策略及食物资源，从而尽量避免种间竞 争。鼠耳蝠属有很多形态相类似且常常共栖一地的 种类，其捕食策略及食物结构却不相同。如大鼠耳 蝠(M. myotis)和尖耳鼠耳蝠(M. blythii), 这两种形态 非常相似的蝙蝠(Arlettaz et al, 1991; Ruedi et al, 1990)常常共栖一地，而食性却大不相同，前者喜欢 在地面取食步甲类昆虫, 后者则喜食蚉斯类 (Arlettaz, 1996, 1999; Arlettaz \& Perrin, 1995; Arlettaz et al, 1991, 1993, 1997)。北美长耳鼠耳蝠 $(M$. evotis) 和北美鼠耳蝠 (M. auriculus) 曾长期被误认为 是同一个种(Genoways \& Jones Jr, 1969), 但食性分 析后发现前者的主要食物为夜蛾类, 后者则喜食甲 虫(Black, 1974)。Begon et al (1996)提出了 “食物资 源分化是动物群落在一定生境共存的关键” 的生态 位理论，可见捕食生态位的分离是不同种蝙蝠，特 别是近缘种能够同地共栖的关键。

水鼠耳蝠 $(M$. daubentonii)过去被认为是广泛分 布于古北界(欧亚大陆)的种类，分布于中国的长期 以来被认为属于水鼠耳蝠, 包括 3 亚种, 分别为 $M$. d. loukashkini、M. d. volgensis 和 M. d. laniger (Wang, 2003)。我国华南地区的 $M$. d . laniger 因体型较小, 前臂长小于 $36 \mathrm{~mm}$, Topal (1997)将其提升为种, 命 名为华南水鼠耳蝠 (M. laniger), 这一观点已被后来 的学者所接受(Pan et al, 2007; Smith et al, 2008; Wang et al, 2010)。毛腿鼠耳蝠(M. fimbriatus)与华南 水鼠耳蝠在外形上非常相似, 为我国特有种; 西南 鼠耳蝠(M. altarium)也属于我国的特有种, 数量较 稀少(Luo et al, 1993)。

Zhou (2001)对毛腿鼠耳蝠夏季的食性进行过 研究, 目前还没有关于华南水鼠耳蝠和西南鼠耳蝠 食性的研究报道。本文对共栖于贵州安龙一山洞中 的华南水鼠耳蝠、毛腿鼠耳蝠和西南鼠耳蝠食性进 行研究和比较, 并结合形态特征的差异和捕食策略, 探讨 3 种鼠耳蝠同地共栖的机制。

\section{1 材料和方法}

野外工作于 2005 年秋季 9-11 月在贵州省安 龙县笃山乡姜家洞(N $25^{\circ} 19^{\prime}$, E $\left.105^{\circ} 05^{\prime}\right)$ 进行。该洞 为一单进口洞, 洞口海拔 $1024 \mathrm{~m}$, 朝西北, 洞口宽 $3 \mathrm{~m}$, 高 $2 \mathrm{~m}$, 洞长约 $1000 \mathrm{~m}$ 。洞内潮湿, 有多处积 水, 多处有石柱、石幔和石钟乳等。该地区属于岩
溶地貌, 海拔 $1000 \sim 1300$ m, 属于南亚热带气候, 年均温较高, 热量条件好, 但降雨量略偏少。生境 主要为呈斑块状分布的常绿阔叶落叶混交林、石山 矮灌丛，以及耕作旱地、水面、灌草丛和竹林。

每隔 2 周采样 1 次, 每次 3 晚, 总计采样 4 次 共计 12 晚。从夜间 20:00一次日 6: 00 在洞口悬 挂雾网, 共捉到回洞的华南水鼠耳蝠 46 只、毛腿鼠 耳蝠 32 只和西南鼠耳蝠 22 只。参考 Luo et al (1993) 鉴定种类、性别, 测量体重(弹簧秤称量, 精确到 $0.1 \mathrm{~g}$ )、前臂长、头体长和耳长(游标卡尺, 精确到 $0.1 \mathrm{~mm})$ 。将蝙蝠装入干净小布袋，每袋一只，并进 行编号。次日清晨收集粪便, 干燥后装入小玻璃瓶 中待分析, 然后将蝙蝠于原洞内释放。我们同时还 捕捉环境中的昆虫, 作为粪便中昆虫残骸鉴定的参 照标本(Whitaker Jr, 1987)。

参考 Kunz \& Parsons (1988)用粪便分析法分析 每种蝙蝠的食物组成。先将粪便浸泡于 $70 \%$ 的异丙 醇中至少 12 h (Belwood \& Fenton, 1976), 然后将粪 便颗粒置于培养血中, 并在培养血下部放一细网篮, 在解剖镜下鉴定粪便中的昆虫残骸，参考郭振中编 著的《贵州农林昆虫志》(Guo et al, 1987), 主要鉴 定到目，对鞘翅目鉴定到科; 同时通过网篮观察统 计各类昆虫所占的体积百分比。所有数据用 SPSS 13.0 for Windows 软件进行处理分析。实验数据用 单因素方差分析 (One-way ANOVA), 以 $P=0.05$ 为检验水准, 统计结果以平均值 \pm 标准误 (Mean $\pm S E$ ) 表示。

\section{2 结 果}

\section{1 形态特征}

经 One-way ANOVA 的 LSD 两两差异显著性 统计分析显示, 3 种鼠耳蝠的体重、头体长、耳长两 两之间差异显著 $(P<0.05)$, 西南鼠耳蝠与另外 2 种 鼠耳蝠的前臂长相比两两之间差异显著 $(P<0.05)$, 华南水鼠耳蝠与毛腿鼠耳蝠的前臂长差异不显著 $(P>0.05) 。 3$ 种鼠耳蝠中, 西南鼠耳蝠的体型最大, 耳最长且向前折超出吻端很多; 华南水鼠耳蝠的体 型最小, 耳长也最短(表 1)。华南水鼠耳蝠和毛腿鼠 耳蝠体型相似，后者在体型上稍大于前者，且胫背 密生线毛，据此可将两者区分。

\section{2 三种鼠耳蝠的食物组成}

每只鼠耳蝠至少分析 5 粒粪便, 共分析粪便 509 粒。结果表明, 3 种蝙蝠皆为食虫性蝙蝠。其 
表 1 三种鼠耳蝠的形态参数对比

Tab. 1 Comparison of morphological data of three Myotis species

\begin{tabular}{|c|c|c|c|}
\hline $\begin{array}{c}\text { 参数 } \\
\text { Parameter }\end{array}$ & $\begin{array}{c}\text { 华南水鼠耳蝠 } \\
\text { Myotis laniger }(n=46)\end{array}$ & $\begin{array}{c}\text { 毛腿鼠耳蝠 } \\
\text { Myotis fimbriatus }(n=32)\end{array}$ & $\begin{array}{c}\text { 西南鼠耳蝠 } \\
\text { Myotis altarium }(n=22)\end{array}$ \\
\hline 体重 Body mass(g) & $4.46 \pm 0.53 \mathrm{a}$ & $5.15 \pm 1.76 b$ & $10.94 \pm 0.87 \mathrm{c}$ \\
\hline 前臂长 Forearm length (mm) & $34.63 \pm 1.45 \mathrm{a}$ & $35.20 \pm 1.07 \mathrm{a}$ & $45.21 \pm 1.15 b$ \\
\hline 头体长 Body length (mm) & $41.06 \pm 2.19 \mathrm{a}$ & $44.43 \pm 1.83 b$ & $53.93 \pm 2.13 \mathrm{c}$ \\
\hline 耳长 Ear length (mm) & $12.00 \pm 1.10 \mathrm{a}$ & $14.21 \pm 0.58 b$ & $20.60 \pm 1.90 c$ \\
\hline
\end{tabular}

注：每一参数中, 不同物种间的不同字母 $(\mathrm{a}, \mathrm{b}, \mathrm{c})$ 表示不同物种之间差异显著 $(P<0.05)$; 相同字母表示不同物种间差异不显著 $(P>0.05)$ 。

Note: For each parameter, different letters $(\mathrm{a}, \mathrm{b}, \mathrm{c})$ indicate a statistical significance $(P<0.05)$; the same letters mean $P>0.05$.

中华南水鼠耳蝠主要捕食近水面活动的双翅目 (Diptera)及其幼虫, 体积百分比和频次百分比分别 为 79.7\% 和 $100 \%$, 其次为小型的鞘翅目(Coleoptera, 主要为金龟子科 Scarabaeidae) 和鳞翅目 (Lepidoptera), 体积百分比分别占 $6.4 \%$ 和 $4.3 \%$, 频 次百分比分别为 $34.9 \%$ 和 $20.3 \%$; 毛腿鼠耳蝠主要 捕食双翅目和小型鞘翅目昆虫 [主要为叶甲科 (Chrysomelidae)和金龟子科(Scarabeidae)], 体积百 分比分别占 $59.6 \%$ 和 $28.8 \%$, 频次百分比分别为 91.3\%和 $80.1 \%$; 西南鼠耳蝠的食物组成主要为近 地面或在地表活动的鞘翅目 [步甲科(Carabidae)和 埋葬虫科(Silphidae)]昆虫, 体积百分比和频次百分 比分别为 $80.8 \%$ 和 $100 \%$ (表 2), 其它目的昆虫体积 百分比总计仅占不到 $20 \%$, 频次百分比也远低于鞘
翅目。对于鳞翅目主要根据粪便中的鳞片来鉴定, 由于鳞翅目昆虫食物存留在肠道内的时间比较长, 有时甚至多达几天, 不能精确估算其粪便中的食物 体积百分比, 但结合频次百分比, 应能较准确反映 其在蝙蝠食物组成中的比例。虽然 3 种蝙蝠的食物 组成有重叠(表 2), 然而经对体积百分比 One-way ANOVA 的 LSD 两两差异显著性统计分析显示, 3 种鼠耳蝠对鞘翅目和双翅目的捕食两两之间差异 显著 $(P<0.05)$; 华南水鼠耳蝠和毛腿鼠耳蝠对毛翅 目的捕食存在显著差异 $(P<0.05)$; 对鳞翅目的捕食, 华南水鼠耳蝠与另外 2 种鼠耳蝠无显著差异 $(P>0.05)$, 毛腿鼠耳蝠与西南鼠耳蝠之间差异显著 $(P<0.05)$ 。

表 2 三种鼠耳蝠的食物组成比较(体积百分比和频次百分比, \%)

Tab. 2 Comparison of each prey category in faecal pellets of three Myotis species (volume percent and frequency percent, \%)

\begin{tabular}{|c|c|c|c|c|c|c|}
\hline \multirow{2}{*}{$\begin{array}{l}\text { 食物组成 } \\
\text { Food items }\end{array}$} & \multicolumn{2}{|c|}{$\begin{array}{c}\text { 华南水鼠耳蝠 } \\
\text { Myotis laniger }(n=232)\end{array}$} & \multicolumn{2}{|c|}{$\begin{array}{c}\text { 毛腿鼠耳蝠 } \\
\text { Myotis fimbriatus }(n=161)\end{array}$} & \multicolumn{2}{|c|}{$\begin{array}{c}\text { 西南鼠耳蝠 } \\
\text { Myotis altarium }(n=116)\end{array}$} \\
\hline & $\begin{array}{c}\text { 体积百分比 } \\
\mathrm{V} \%\end{array}$ & $\begin{array}{c}\text { 频次百分比 } \\
\mathrm{F} \%\end{array}$ & $\begin{array}{c}\text { 体积百分比 } \\
\mathrm{V} \%\end{array}$ & $\begin{array}{c}\text { 频次百分比 } \\
\mathrm{F} \%\end{array}$ & $\begin{array}{c}\text { 体积百分比 } \\
\mathrm{V} \%\end{array}$ & $\begin{array}{c}\text { 频次百分比 } \\
\mathrm{F} \%\end{array}$ \\
\hline 鞘翅目 Coleoptera & $6.4 \pm 5.0 \mathrm{a}$ & 34.9 & $28.8 \pm 11.2 \mathrm{~b}$ & 80.1 & $80.8 \pm 14.4 \mathrm{c}$ & 100.0 \\
\hline 鳞翅目 Lepidoptera & $4.3 \pm 3.7 \mathrm{ab}$ & 20.3 & $4.8 \pm 4.5 \mathrm{a}$ & 26.1 & $3.5 \pm 2.8 \mathrm{~b}$ & 15.5 \\
\hline 双翅目 Diptera & $79.7 \pm 16.8 \mathrm{a}$ & 100.0 & $59.6 \pm 13.8 b$ & 91.3 & $1.6 \pm 1.7 \mathrm{c}$ & 9.5 \\
\hline 直翅目 Orthoptera & 0 & 0 & 0 & 0 & $2.9 \pm 2.8$ & 5.2 \\
\hline 脉翅目 Neuroptera & 0 & 0 & 0 & 0 & $1.0 \pm 0.9$ & 3.4 \\
\hline 毛翅目 Trichoptera & $1.9 \pm 2 . .2 \mathrm{a}$ & 8.2 & $2.9 \pm 3.4 \mathrm{~b}$ & 12.4 & 0 & 0 \\
\hline 未鉴定 Not identified & $7.5 \pm 3.0$ & & $3.8 \pm 2.2$ & & $9.7 \pm 3.3$ & \\
\hline
\end{tabular}

每一参数中不同物种间的不同字母含义同表 1 。

For each parameter, different letters among species is the same as shown in Tab.1.

\section{3 讨 论}

同地共栖的动物物种间为避免彼此间的激烈 竞争至少要在某一维的生态位上利用不同的资源
(Siemers \& Schnitzler, 2004)。尽管这种行为是由进 化因素还是生态因素引起的目前还存在争议, 但至 少在这一点上存在共识: 这种行为一旦建立, 即会 引起资源的分割, 使得不同的物种能够共存, 从而 
促进了物种多样性(Fox, 2004)。Arlettaz et al (2000) 认为食物资源竞争影响蝙蝠的种群格局, 同一山洞 的几种蝙蝠种间竞争明显(Gause, 1934), 它们能分 享栖息环境应与生态位分化不可分割 (Swift \& Racey, 1983)。因此, 捕食生态位分离是多种蝙蝠同 地共栖的关键因素之一(Arlettaz, 1999)。

华南水鼠耳蝠和毛腿鼠耳蝠形态上非常接近, 我们此次通过对同居一地的两种蝙蝠的形态、食物 组成的研究发现, 两者存在显著差异。Liu et al (2003)结合回声定位叫声的声波特点分析推断毛腿 鼠耳幅多在不太复杂的近距离环境中捕食, 并且发 现其食物中双翅目的昆虫的比例高达 $87 \%$ 。本研究 发现, 毛腿鼠耳蝠和华南水鼠耳蝠均捕食相当数量 的双翅目, 但华南水鼠耳蝠食物组成中双翅目显著 多于毛腿鼠耳蝠。由于条件所限, 本研究没有对蝙 蝠的捕食行为进行跟踪研究, 但本研究中华南水鼠 耳蝠的食物中有相当多是靠近水面活动的双翅目 幼虫; 而早前对同一地区毛腿鼠耳蝠的研究发现其 主要是在旱地上空捕食(Zhou, 2001), 说明两者虽 然都捕食双翅目昆虫，但其捕食空间发生了分化。

许多研究表明, 很多近缘物种形态的差异造成 它们食物的差异(Schluter, 2000)。与毛腿鼠耳蝠相 比, 西南鼠耳蝠体型较大且有着相对较长的耳朵。

Fenton \& Bogdnowicz (2002)研究证实, 近地面或在 地面的掠食者耳较长、体型较大, 采用拾遗式 (Gleaning)捕食策略。通过对一些耳长较大的种类, 如纳氏鼠耳蝠(M. nattereri) (Swift \& Racey, 2002)、 北美长耳鼠耳蝠(M. evotis) (Faure \& Barclay, 1992) 的研究表明, 它们的回声定位用于在飞行中导航, 而对猎物的定位主要通过辨别猎物翅膀的震动或 者猎物发出的声音来完成, 能够捕捉到躲藏在植被

\section{参考文献:}

Altringham JD, McOwat T, Hammond L. 1998. Bats: Biology and Behaviour [M]. Oxford: Oxford University Press, USA.

Arlettaz R. 1996. Feeding behaviour and foraging strategy of free-living mouse-eared bats, Myotis myotis and Myotis blythii [J]. Anim Behav, 51(1):1-11.

Arlettaz R. 1999. Habitat selection as a major resource partitioning mechanism between the two sympatric sibling bat species Myotis myotis and Myotis blythii [J]. J Anim Ecol, 68(3):460-471.

Arlettaz R, Godat S, Meyer H. 2000. Competition for food by expanding pipistrelle bat populations (Pipistrellus pipistrellus) might contribute to the decline of lesser horseshoe bats (Rhinolophus hipposideros) [J]. Biol Conserv, 93(1):55-60.

Arlettaz R, Perrin N. 1995. The trophic niches of sympatric sibling Myotis
中或近地面等背景噪声复杂的环境中的昆虫。我们 的研究发现, 虽然毛腿鼠耳蝠和西南鼠耳蝠都捕食 大量的鞘翅目昆虫, 但西南鼠耳蝠捕食的多为近地 面或在地表活动的步甲科和埋葬虫科的昆虫，而毛 腿鼠耳蝠捕食的主要为在空中飞行的小型鞘翅目 昆虫，其捕食空间发生了分化。同时两者食物组成 中的鞘翅目差异显著，西南鼠耳蝠食物中的鞘翅目 显著多于毛腿鼠耳蝠。

周江于 1999 年和 2000 年夏季对同一地区毛腿 鼠耳蝠的食性进行了研究(Zhou, 2001), 发现在其 食物组成中，双翅目的体积百分比，占 $83.8 \%$, 而鞘 翅目, 仅占 $3.1 \%$; 我们此次对其食性的研究结果发 现，鞘翅目的体积百分比达 $28.8 \%$, 而双翅目, 占 $59.6 \%$ 。Altringham et al (1998)认为蝙蝠的食物组成 和季节变化与环境中食物的丰富度有关, 如 Ma et al (2005)通过对北京房山地区的大足鼠耳蝠两年的粪 便样品进行分析后发现，两年间的食物成分含量差 异显著。由此可推测, 毛腿鼠耳蝠的食物组成也可 能由于不同的季节或年份而有所波动。

食物资源分化是蝙蝠在一定生境中共存的关键 (Begon et al, 1996)。本研究中由粪便分析结果可以看 出, 同一山洞的 3 种鼠耳蝠的食物组成虽有重叠, 但 存在相当大的差异，且捕食空间发生分化，因此，其 生态位表现出明显的分化。这使得 3 个物种共存于 同一栖息地，避免了对食物资源和捕食空间的竞争。

致谢: 蝙蝠鉴定承蒙贵州师范大学谷晓明教 授、黎道洪教授的帮助和指点; 粪便分析和昆虫鉴 定得到贵州师范大学罗庆怀教授的帮助和指导; 野 外工作得到游太勇、张竹青、罗卫东同学的协助，在 此一并致谢。

myotis and M. blythii: do mouse-eared bats select prey [J]? Symp Zool Soc, London, 67:361-376.

Arlettaz R, Ruedi M, Hausser J. 1991. Field morphological identification of Myotis myotis and Myotis blythii (Chiroptera, Vespertilionidae): a multivariate approach [J]. Myotis, 29(7):16.

Arlettaz R, Ruedi M, Hausser J. 1993. Ecologie trophique de deux espèces jumelles et sympatriques de chauves-souris: Myotis myotis et Myotis blythii (Chiroptera: Vespertilionidae). Premiers résultats [J]. Mammalia, 57(4):519-532.

Arlettaz R, Ruedi M, Ibanez C, Palmeirim J, Hausser J. 1997. A new perspective on the zoogeography of the sibling mouse-eared bat species Myotis myotis and Myotis blythii: morphological, genetical and ecological evidence [J]. J Zool, 242(1):45-62. 
Begon M, Harper JL, Townsend CR. 1996. Ecology: individuals, populations, and communities [M]. Oxford: Blackwell Scientific Publications.

Belwood JJ, Fenton MB. 1976. Variation in the diet of Myotis lucifugus (Chiroptera: Vespertilionidae) [M]. Can J Zool, 54(10):1674-1678.

Black HL. 1974. A north temperate bat community: structure and prey populations [J]. J Mammal, 55(1):138-157.

Faure PA, Barclay RMR. 1992. The sensory basis of prey detection by the long-eared bat, Myotis evotis, and the consequences for prey selection [J]. Anim Behav, 44(1):31-39.

Fenton MB, Bogdanowicz W. 2002. Relationships between external morphology and foraging behaviour: bats in the genus Myotis [J]. Can J Zool, 80:1004-1013.

Fox JW. 2004. Modelling the joint effects of predator and prey diversity on total prey biomass [J]. J Anim Ecol, 73(1):88-96.

Gaisler J, Bauerova Z. 1985. The life of bats in a city [J]. Myotis, 23(24):209-215.

Gause GF. 1934. The Struggle for Existence [M]. New York: Hafner.

Genoways HH, Jones Jr JK. 1969. Taxonomic status of certain long-eared bats (genus Myotis) from the Southwestern United States and Mexico [J]. Southwest Nat, 14(1):1-13.

Guo ZZ, Zheng ZM, Chen FY. 1987. Agriculture Insect Fauna in Guizhou [M]. Guiyang: Guizhou People's Publishing House. [郭振中. 1987. 贵 州农林昆虫志 $[\mathrm{M}]$. 贵阳: 贵州人民出版社.]

Kunz TH, Parsons S. 1988. Ecological and Behavioral Methods for theStudy of Bats [M]. Washington, DC: Smithsonian Institution Press, 7-80 .

Liu Y, Feng J, Chen M, Zhao HH, Zhou J, Zhang SY. 2003. The analysis on echolocation calls of Myotis fimbriatus (Chiroptera: Vespertilionidae) [J]. J Northeast Normal Univ: Nat Ed. 35(2):113-116. [刘颖, 冯江, 陈 敏, 赵辉华, 周江, 张树义. 2003. 毛腿鼠耳蝠回声定位声波的分析 [J]. 东北师大学报: 自然科学版, 35(2):113-116.]

Luo R, Xie JH, Gu YH, Li DH. 1993. The Mammalian Fauna of Guizhou [M]. Guiyang: Guizhou People's Publishing House.[罗蓉, 谢家骅, 辜 永河, 黎道洪. 1993. 贵州兽类志[M]. 贵阳: 贵州人民出版社.]

Ma J, Jones G, Zhang S, Shen J, Metzner W, Zhang L, Liang B. 2003. Dietary analysis confirms that Rickett's big-footed bat (Myotis ricketti) is a piscivore [J]. $J$ Zool, 261(3):245-248.

Ma J, Liang B, Zhang JS, Zhang JP, Zhang SY. 2005. The major item and seasonal variation in the diet of Rickett's big-footed bat Myotis ricketti in Beijing [J]. Acta Zool Sin, 51(1):7-11.[马杰, 梁冰, 张劲硕, 张俊 鹏, 张树义. 2005. 北京地区大足鼠耳蝠主要食物及其食性组成的 季节变化[J]. 动物学报, 51(1): 7-11.]

Pan QH, Wang YX, Yan K. 2007. A Field Guide to the Mammals of China [M]. Beijing: China Forestry Publishing House. [潘清华, 王应祥, 岩 芘. 2007. 中国哺乳动物彩色图鉴[M]. 北京: 中国林业出版社.]

Ruedi M, Arlettaz R, Maddalena T. 1990. Distinction morphologique et biochimique de deux especes jumelles de chauves-souris: Myotis myotis (Bork.) et Myotis blythi (Tomes)(Mammalia; Vespertilionidae) [J]. Mammalia, 54(3):415-430.

Schluter D. 2000. Ecology of Adaptive Radiation [M]. Oxford: Oxford University Press.

Siemers BM, Schnitzler HU. 2004. Echolocation signals reflect niche differentiation in five sympatric congeneric bat species [J]. Nature, 429(6992):657-661.

Smith AT, Xie Y, Hoffmann RS, Lunde D, MacKinnon J, Wilson DE, Wozencraft WC. 2008. A Guide to the Mammals of China [M]. Princeton: Princeton University Press, 375.

Topal G. 1997. A new mouse-eared bat species, from Nepal, with statistical analyses of some other species of subgenus Leuconoe (Chiroptera, Vespertilionidae) [J]. Acta Zool Hung, 43(4): 375-402.

Wang L, Jiang TL, Sun KP, Wang YX, Tiunov MP, Feng J. 2010. Morphological description and taxonomical status of Myotis petax [J]. Acta Zootax Sin, 35(2): 360-365. [王否, 江廷磊, 孙克萍, 王应祥, Tiunov MP, 冯江. 2010. 东亚水鼠耳蝠形态描述与分类 $[\mathrm{J}]$. 动物分 类学报, 35(2): 360-365.]

Wang YX. 2003. A Complete Checklist of Mammal Species and Subspecies in China: a Taxonomic and Geographic Reference [M]. Beijing: China Forestry Publishing House, 41-46. [王应祥. 2003. 中国哺乳动物种和 亚种分类名录与分布大全[M]. 北京: 中国林业出版社, 41-46.]

Whitaker Jr JO. 1987. Food habits analysis of insectivorous bats [M]//Kunz TH, ed. Ecological and Behavioral Methods for the Study of Bats. Washington, DC: Smithsonian Institution Press, 171-189.

Zhou J. 2001. Spatial distributing patterns and intra-species relationships of seven bats in Guizhou Province [D]. M.s. Thesis. Guiyang: Guizhou Normal Univ. [周江. 2001. 贵州省七种蝙蝠空间生态位及种间关系 研究[D]. 硕士论文. 贵阳：贵州师范大学.] 